\section{Sandrine Barbaux Éric Vilain Ken McElreavey Marc Fellous}

\author{
ADRESSE \\ $\mathrm{S}$. Barbaux : étudiante en doctorat, boursière \\ $M R E$ E. Vilain : docteur ès sciences. $\mathrm{K}$ McEl- \\ reavey : chargé de recherche à l'Institut Pasteur. \\ M. Fellous : professeur d'immunogénétique hu- \\ maine. Laboratoire d'immunogenétique hu- \\ maine, Inserm U 276, Institut Pasteur, 25, \\ rue du Docteur-Roux, 75724 Paris Cedex \\ 15 , France.
}

\title{
Le point sur le déterminisme du sexe chez les mammifères
}

Le déterminisme du sexe est l'ensemble des mécanismes conduisant une gonade indifférenciée à s'engager dans une voie de développement, soit testiculaire, soit ovarienne. Il s'agit d'une cascade d'événements très complexe qui nécessite l'interaction de nombreux gènes. Le gène $S R Y$ (sex determining region, Y chromosome) a été identifié au TDF (testis determining factor) qui est le signal primaire déclenchant la formation du testicule. Grâce à l'analyse de sujets avec une réversion du sexe et de modèles animaux, le rôle de certains gènes dans la détermination du sexe a pu être démontré. Cependant, de nombreuses étapes de cette cascade restent encore inconnues.

e déterminisme du sexe est l'ensemble des mécanismes conduisant une gonade foetale indifférenciée à s'engager dans une voie de développement mâle ou femelle pour aboutir à un testicule ou un ovaire. Chez les mammifères, le contrôle de la détermination du sexe est d'ordre génétique. Le chromosome $\mathrm{Y}$ joue un rôle dominant dans le déterminisme du testicule. Indépendamment du nombre de chromosomes $\mathrm{X}$, un individu porteur d'un chromosome $\mathrm{Y}$ se développe dans le sens mâle : les patients 47,XXY (syndrome de Klinefelter) ont un phénotype essentiellement masculin alors que les individus 45, XO atteints du syndrome de Turner présentent un phénotype féminin. Dans d'autres espèces, le déterminisme du sexe est soumis à d'autres contrôles. Chez Drosophila melanogaster et Caenorhabditis elegans par exemple, le signal primaire est le rapport du nombre de chromosome(s) X au nombre d'autosomes. Ce signal peut également dépendre de facteurs liés à l'environnement, com- me dans le cas de certains reptiles où le sexe dépend de la température d'incubation des œufs.

La notion de déterminisme se distingue de celle de différenciation sexuelle. La différenciation sexuelle englobe les processus permettant, une fois la gonade formée, la réalisation d'un phénotype sexuel (organes génitaux internes et externes). La frontière entre ces deux notions est en fait artificielle car il s'agit d'un enchaînement continu d'événements au cours du développement. Chez les mammifères, les expériences du PrJost ont montré que le testicule avait un rôle dominant dans la différenciation sexuelle [1]. En effet, la présence d'un testicule permet le développement d'un individu mâle alors que l'absence de testicule ou la présence d'un ovaire donnent naissance à un individu femelle. La différenciation sexuelle mâle se réalise sous l'influence d'hormones produites par le testicule foetal, la testostérone (et son dérivé la dihydrotestostérone) et l'hormone antimüllérienne (ou $\mathrm{AMH}$ ). 


\section{RÉFÉRENCES}

1. Jost A. Les péripéties d'une recherche: l'étude de la différenciation sexuelle. médecine/sciences $1991 ; 7$ : 263-75.

2. Bogan JS, Page DC. Ovary? Testis?: a mammalian dilemma. Cell $1994 ; 76: 603-7$.

3. Vilain E, McElreavey K, Herskowitz I, Fellous $\mathrm{M}$. La détermination du sexe : faits et nouveaux concepts. médecine/sciences 1992 $8: 1-7$

4. Gubbay J, Collignon J, Koopman P, Capel B, Economou A, Münsterberg A, Vivian N, Goodfellow PN, Lovell-Badge R. A gene mapping to the sex-determining region of the mouse $Y$ chromosome is a member of a novel family of embryonically expressed genes. Nature 1990 ; 346 : 245-50.

5. Sinclair AH, Berta P, Palmer MS, Hawkins JR, Griffiths BL, Smith MJ, Foster JW, Frischauf AM, Lovell-Badge R, Goodfellow PN. A gene from the human sex-determining region encodes a protein with homology to a conserved DNA-binding motif. $\mathrm{Na}$ ture $1990 ; 346: 240-5$.

6. Koopman P, Gubbay J, Vivian N, Goodfellow PN, Lovell-Badge $R$. Male development of chromosomally female mice transgenic for Sry. Nature 1991 ; 351 : 117-21.

7. Burgoyne PS, Mahadevaiah SK, Sutcliffe $\mathrm{MJ}$, Palmer SJ. Fertility in mice requires X-Y pairing and a Y-chromosomal «spermiogenesis" gene mapping to the long arm. Cell nesis" gene mappin

8. Ma K, Sharkey A, Kirsch S, Vogt P, Keil R, Hargreave TB, McBeath S, Chandley AC. Towards the molecular localisation of the AZF locus: mapping of microdeletions in azoospermic men within 14 subintervals of interval 6 of the human $Y$ chromosome. Hum Mol Genet 1992 ; 1 : 29-33.

9. Hagq CM, King CY, Donahoe PK, Weiss MA. $S R Y$ recognizes conserved DNA sites in sex-specific promoters. Proc Nall Acad Sci USA 1993 ; 90 : 1097-101.

10. Giese K, Pagel J, Grosschedl R. Distinct DNA-binding properties of the high mobility group domain of murine and human $S R Y$ sex-determining factors. Proc Natl Acad Sci USA $1994 ; 91$ : 3368-72.

11. Capel B, Swain A, Nicolis S, Hacker A Walter M, Koopman P, Goodfellow PN, Lovell-Badge $R$. Circular transcripts of the testis-determining gene $S r y$ in adult mouse testis. Cell $1993 ; 73: 1019-30$.

12. Clépet C, Schafer AI, Sinclair AH, Palmer MS, Lovell-Badge $R$, Goodfellow PN. The human SRY transcript. Hum Mol Genet $1993 ; 2$ : 2007-12.

13. McElreavey K, Vilain E, Abbas N, Costa JM, Souleyreau N, Kucheria K, Boucekkine C, Thibaud E, Brauner R, Flamant F, Fellous M. XY sex reversal associated with a deletion 5' to the $S R Y$ "HMG box " in the testis-determining region. Proc Natl Acad Sci
Les données actuelles recueillies sur le déterminisme du sexe restent encore limitées. Le facteur primaire initiateur de la cascade de détermination du testicule porté par le chromosome $\mathrm{Y}$, nommé TDF pour testis determining factor chez l'homme et Tdy chez la souris, a été identifié et cloné. Néanmoins, cette cascade complexe de développement aboutissant à un individu masculin ou féminin comprend de nombreuses autres étapes qui échappent encore à notre compréhension [2]. La connaissance de ces mécanismes nous permettra de connaître les causes des maladies de réversion du sexe, qui sont graves tant sur le plan clinique que psychologique. Ces maladies, détaillées dans le Tableau I, sont probablement dues à des mutations dans les gènes du déterminisme du sexe et leur étude a permis, et permet encore, de progresser dans la connaissance des bases moléculaires du déterminisme et de la différenciation des gonades. Cette revue a pour but de faire le point sur les informations collectées sur le facteur TDF, codé par le gène $S R Y$, ainsi que sur les recherches mises en œuvre pour identifier de nouveaux facteurs agissant tout au long de la cascade.

\section{Le point sur SRY}

\section{SRY est le testis determining factor}

Après de nombreuses années de recherche à la quête du TDF, et l'abandon de plusieurs gènes candidats tels que $H Y$ et $Z F Y$ [3], les gènes $S R Y$ (humain) et sry (murin) ont finalement

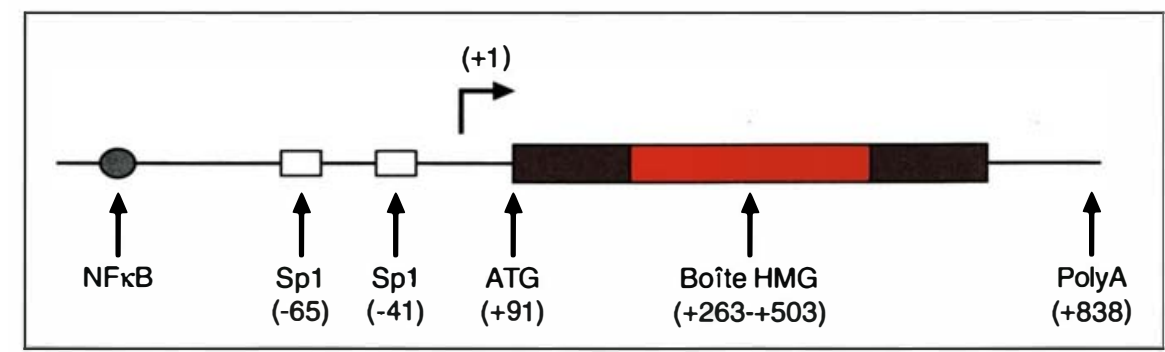

Figure 1. Schéma de la structure du gène SRY humain. Des séquences consensus de fixation des facteurs de transcription $N F-\kappa B$ (facteur de transcription inductible par différents signaux et contrôlant l'activité de nombreux gènes et virus) et Sp1 ont été identifiées dans la région proximale en $5^{\prime}$ du gène SRY humain. La flèche notée +1 correspond au site majeur de début de la transcription. La boîte colorée en rouge représente le domaine HMG de fixation à I'ADN. L'abréviation polyA désigne le site de polyadénylation. été clonés en $1990[4,5]$. Une série d'éléments montre que SRY est bien le signal primaire de la détermination testiculaire: (1) le gène $S R Y$ est spécifique du chromosome Y qui joue un rôle dominant dans le déterminisme du testicule, indépendamment du nombre de chromosome(s) les mammiferes un fragment de $35 \mathrm{~kb}$ d'ADN localisé dans la partie terminale du bras court du chromosome $\mathrm{Y}$, caractérisé comme la région minimale permettant une réversion de sexe. Cet interalle a pu être défini grâce à l'analyse d'une large collection de patients, des femmes XY porteuses d'une déléhette région d'ADN et des cation de cette région ; (3) l'étude du profil d'expression de $S R Y$ est en accord avec la fonction suggérée du TDF. Des ARN messagers de sry sont détectés au niveau de la gonade fotale de souris au moment précis où une différenciation testiculaire peut être observée par l'apparition des de Sertoli (à partir de 10,5 jours de développement chez la souris, 29 jours chez le mouton et vers la sixième semaine chez l'homme) ; (4) l'étude de femmes XY présentant une dysgénésie gonadique, c'est-àdire des gonades dépourvues d'ovocytes et constituées uniquement de tissus fibreux, a permis la découverte de mutations ponctuelles de novo dans la phase ouverte de lecture du gène $S R Y$ et, plus précisément, dans le domaine de liaison à l'ADN de la protéine; (5) enfin, l'argument le

$$
m / s n^{\circ} 4, \text { vol. } 11 \text {, avril } 95
$$


plus fort a été apporté par des expériences de transgénèse, introduisant un fragment de $14 \mathrm{~kb}$ d'ADN contenant le gène sry dans des embryons de souris de caryotype XX. L'expression du transgène sry induit une réversion du sexe et un développement dans le sens mâle de ces souris [6]. Il est intéressant de noter que ces souris mâles $\mathrm{XX}$, tout comme les hommes XX, sont stériles. A l'âge adulte, leurs gonades sont dépourvues de cellules germinales, alors qu'à des stades plus précoces ces dernières peuvent encore être observées. Plusieurs hypothèses peuvent être formulées. La disparition de ces cellules germinales pourrait être due à l'absence, pendant la méiose, d'un appariement $\mathrm{X}-\mathrm{Y}$ qui semble être indispensable pour la spermiogenèse de souris [7]. La survie des spermato- zoïdes semble également dépendre d'un facteur nommé AZF pour azoospermia factor, localisé sur le bras long du chromosome $\mathrm{Y}$ et absent chez les mâles XX [8].

\section{Organisation génique de SRY}

$S R Y$ est un gène situé dans la partie terminale du bras court du chromosome $\mathrm{Y}$, à proximité de la région pseudoautosomique. Ce gène, constitué d'un unique exon, est composé d'une phase ouverte de lecture de 669 paires de bases (figure 1). Il code pour une protéine longue de 223 acides aminés qui possède un domaine central conservé par rapport à son homologue murin et dans d'autres espèces (figure 2). Cette boîte de 80 acides aminés, baptisée HMG à cause de son homologie avec les protéines du high mobility group entrant dans la composition de la chromatine, confère à la protéine SRY des propriétés de liaison à l'ADN. Outre sa capacité de fixation à de courtes séquences spécifiques [9], la protéine SRY a la possibilité de se lier à l'ADN cruciforme et de courber l'ADN $\left(m / s n^{\circ} 2\right.$, vol. 8, p. 190) [10].

Les transcrits de sry sont retrouvés au niveau de la crête génitale entre 10,5 et 12,5 jours post coitum chez la souris, au moment précis de l'apparition des premières cellules de Sertoli. L'hypothèse la plus probable est que sry s'exprime de manière autonome dans des cellules "pré-Sertoli», induisant ainsi leur différenciation en cellules de Sertoli. Des communications intercellulaires (avec les futures cellules myoïdes péritubulaires)

Tableau I

LES PRINCIPALES ANOMALIES DE LA DÉTERMINATION DU SEXE CHEZ L'HOMME

\begin{tabular}{|c|c|c|c|c|}
\hline & $\begin{array}{c}\text { Mâle XX } \\
\text { sans ambiguïtés }\end{array}$ & $\begin{array}{c}\text { Mâle XX } \\
\text { avec ambiguïtés }\end{array}$ & $\begin{array}{c}\text { Mâle XX } \\
\text { hermaphrodite }\end{array}$ & $\begin{array}{c}\text { Femme } X Y \text { avec } \\
\text { dysgénésie gonadique }\end{array}$ \\
\hline Fréquence par naissance & $1 / 20000$ & $1 / 20000$ & $1 / 20000$ & très rare \\
\hline Cytogénétique & $46, X X$ & $46, X X$ & $46, X X$ & $46, X Y$ \\
\hline Gonades & $\begin{array}{l}\text { testicule sans } \\
\text { cellules germinales }\end{array}$ & $\begin{array}{c}\text { testicule sans } \\
\text { cellules germinales }\end{array}$ & $\begin{array}{l}\text { tissu testiculaire } \\
\text { et ovarien }\end{array}$ & $\begin{array}{l}\text { gonade dysgénétique } \\
\text { en bandelettes fibreuses }\end{array}$ \\
\hline $\begin{array}{l}\text { Organes génitaux } \\
\text { externes }\end{array}$ & mâle normal & $\begin{array}{c}\text { ambiguïtés génitales } \\
\text { micropénis, } \\
\text { hypospadias }\end{array}$ & ambiguïtés génitales & femmes normales \\
\hline Clinique & stérilité & ambiguïté sexuelle & $\begin{array}{l}\text { ambiguïté sexuelle } \\
\text { gonadoblastome }\end{array}$ & aménorrhée, stérilité \\
\hline
\end{tabular}

Humain DRVKRPMNAFIVWSRDQRRKMALENPRMRNSEISKQLGYQWKMLTEAEKWPFFQEAQKLQAMHREKYPNYKYRPRRKAK 100 Lapin ERVKRPMNAFMVWSQHQRRQVALENPKMRNSDISKQLGHQWKMLSEAEKWPFFQEAQRLQAMHKEKYPDYKYRPRRKVK 82

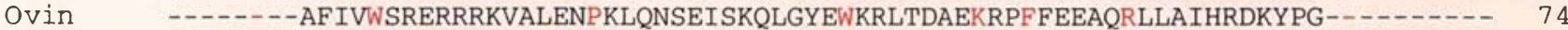

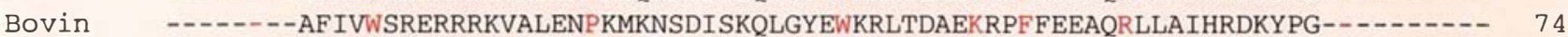

Souris GHVKRPMNAFMVWSRGERHKLAQQNPSMQNTEISKQLGCRWKSLTEAEKRPFFQEAQRLKILHREKYPNYKYQPHRRAK 71

S. macrO SRVKRPMNAFMVWSQTQRRKVALQNPKMHNSEISKQLGVTWKLLSDSEKRPF IDEAKRLRDKHKOV-SDYKYQPRRKTK 59

Figure 2. Comparaison des motifs HMG des gènes SRV de différentes espèces de mammifères. Le pourcentage d'analogie des séquences est calculé par rapport à la protéine SRY humaine. Les boîtes HMG des gènes SRY de mammifères clonés incluent celles d'homme, de lapin, d'ovin et de bovin [37], de souris et de Sminthopsis macroura. Les résidus particulièrement conservés entre les espèces apparaissent en rouge.

$\mathrm{m} / \mathrm{s} n^{\circ} 4$, vol.11, avril 95 


\section{RÉFÉRENCES}

14. McElreavey K, Vilain E, Abbas N, Herskowitz I, Fellous M. A regulatory cascade hypothesis for mammalian sex determination: $S R Y$ represses a negative regulator of male development. Proc Natl Acad Sci USA 1993 ; development
$90: 3368-72$

15. Pritchard-Jones K, Fleming S, Davidson D, Bickmore W, Gosden C, Bard J, Buckler A, Pelletier J, Housman D, van Heyningen V, Hastie N. The candidate Wilms' tumour gene is involved in genitourinary development. Nature $1990 ; 346: 194-7$.

16. Junien C. Un gène candidat pour la tumeur de Wilms. médecine/sciences $1990 ; 6$ : 464-9.

17. Kreidberg JA, Sariola H, Loring JM, Maeda M, Pelletier J, Housman D, Jaenisch R. WT- 1 is required for early kidney development. Cell $1993 ; 74$; 679-91.

18. Lavorgna G, Ueda H, Clos J, Wu C. FTZ$\mathrm{F} 1$, a steroid hormone receptor-like protein implicated in the activation of fushi taratzu. Science 1991 ; 252 : 848-51.

19. Ikeda Y, Lala DS, Luo X, Kim E, Moisan MP, Parker KL. Characterization of the mouse $F T Z F 1$ gene, which encodes a key regulator of steroid hydroxylase gene expression. Mol Endocrinol 1993 ; 7 : 852-60.

20. Shen WH, Moore CCD, Ikeda Y, Parker KL, Ingraham HA. Nuclear receptor steroidogenic factor 1 regulates the Mullerian inhibiting substance gene: a link to the sex determination cascade. Cell $1994 ; 77: 651$ 61.

21. Luo X, Ikeda Y, Parker KL. A cell-specific nuclear receptor is essential for adrenal and gonadal development and sexual differentiation. Cell 1994; 77 : 481-90.

22. Saez IM, Durand P. Rôle du facteur SF-1 dans le développement des gonades et des surrénales, et dans la stéroïdogenèse. méde cine/sciences $1994 ; 10: 1315-7$.

23. Josso N, Picard JY, Vigier B, Tran D. L'hormone antimüllerienne. médecine/sciences $1987 ; 3: 44452$

24. Münsterberg A, Lovell-Badge R. Expression of the mouse anti-Müllerian hormone gene suggests a role in both male and female sexual differenciation. Development 1991 ; $113: 613-24$.

25. Imbeaud S, Carré-Eusèbe D, Rey R, Belville C, Josso N, Picard JY. Molecular genetics of the persistent Müllerian duct syndrome: a study of 19 families. Hum Mol Genet $1994 ; 3: 125-31$.

26. Behringer R, Cate RL, Froelick GI, Pal miter R, Brinster R. Abnormal sexual development in transgenic mice chronically ex pressing Müllerian inhibiting substance. Nature $1990 ; 345: 167-70$

27. Cooke CT, Mulcahy MT, Cullity GJ, Watson $M$, Srague $\mathbf{P}$. Campomyelic dysplasia with sex reversal: morphological and cytogenetic studies of a case. Pathology $1985 ; 17$ pourraient également intervenir dans ce processus. Les transcrits de $S R Y$ sont aussi présents dans le testicule adulte humain, ainsi que chez la souris où ils adoptent une forme particulière circulaire [11]. Le rôle de SRY dans le testicule adulte n'est pas connu. Des messagers ont également été détectés dans de nombreux organes humains (foie, rein, cœur, tissus fotaux) grâce à des méthodes de transcription inverse et de PCR, mais la méthodologie employée peut faire douter de la réalité physiologique de la présence de ces transcrits [12]. La spécificité spatiale et temporelle de l'expresssion de $S R Y$ implique des mécanismes précis de régulation. Ainsi, l'étude du promoteur du gène $S R Y$ qui est en cours a pour but d'identifier les facteurs assurant cette régulation. L'analyse de la région du gène $S R Y$ chez une patiente $\mathrm{XY}$ atteinte de dysgénésie gonadique partielle a permis de découvrir une délé tion, préservant la phase ouverte de lecture de $S R Y$, mais emportant un fragment d'ADN d'au moins $33 \mathrm{~kb}$ en amont du gène $\left(\mathrm{m} / \mathrm{s} n^{\circ} 1, v o l .10\right.$, p. 120) [13]. Ce cas montre l'importance de l'étude des régions régulatrices de $S R Y$.

\section{Rôle de SRY}

La localisation nucléaire de la protéine SRY ainsi que ses propriétés de liaison et de courbure de l'ADN suggèrent fortement un rôle de facteur de transcription $\left(m / s \quad n^{\circ} 2\right.$, vol. 8 , p. 190). Tant que la ou les cible(s) directes de SRY demeurent inconnues, le mode d'action exact de la protéine ne peut être clairement défini. Néanmoins, le gène $S R Y$ n'est pas suffisant pour expliquer tous les cas d'ambiguïtés sexuelles décrits. En effet, seules $20 \%$ des femmes XY présentant une dysgénésie gonadique possèdent une mutation dans leur gène $S R Y$. De même, la présence de $S R Y$ (résultat d'un crossing-over inégal lors de la méiose paternelle) ne peut être détectée que chez $80 \%$ des hommes $\mathrm{XX}$ et $10 \%$ des hermaphrodites vrais XX [14]. Ces résultats imposent l'idée que d'autres gènes sont impliqués dans la cascade de détermination du sexe, différents de $S R Y$ et encore inconnus. Toutefois, l'étude de tous les patients atteints de réversion sexuelle et, en particulier, des rares cas familiaux, permet de formuler des hypothèses. L'existence de frères de caryotype XX (non porteurs de $S R Y$ ), avec un phénotype masculin et nés de parents normaux, suggère l'hypothèse simple d'une mutation dans un gène autre que $S R Y$, se transmettant dans la famille sur le mode autosomique récessif. Selon cette hypothèse, un modèle a été proposé ayant pour but d'expliquer les maladies humaines du déterminisme du sexe [14]. Supposons l'existence d'un gène $Z$, agissant comme répresseur des gènes de la voie de développement masculine et que $Z$ soit la cible de $S R Y$ qui agit de façon négative sur Z, soit par répression de sa synthèse, soit par inhibition ou compétition. Il est alors simple de montrer que chez un homme normal ou un homme XX porteur de $S R Y, S R Y$ inhibe $Z$, permettant ainsi une activation des gènes spécifiques du sexe masculin (figure 3A). En revanche, chez une femme normale, ou $\mathrm{XY}$ avec une délétion de $S R Y, Z$ peut réprimer la voie masculine en l'absence de $S R Y$ (figure 3B). Selon ce modèle, toute variation de la fonction ou de la spécificité de $Z$ doit avoir des répercussions dramatiques sur le phénotype des individus porteurs de mutations. Ainsi, une perte de fonction totale ou partielle de $Z$ peut expliquer l'apparition d'un phénotype masculin plus ou moins ambigu chez un individu $\mathrm{XX}$ (figure 3C). $\mathrm{Au}$ contraire, si une mutation affecte la relation $S R Y-Z$ pour rendre $Z$ indépendant ou insensible à l'action de $S R Y$, l'effet dominant du chromosome Y sur la détermination du testicule est court-circuité (mutation $Z, f i$ gure $3 D$ ). Les récentes données désignant la région Xp21-22 comme importante dans la détermination sexuelle (voir plus bas) sont compatibles avec ce modèle. Une duplication d'une région critique en Xp2122 aboutit en effet à une réversion sexuelle. Il est possible que $Z$ soit localisé dans cette région et que, étant en double dose, il ne puisse subir une complète répression par $S R Y$. Cela conduirait à une activation seulement partielle des gènes spécifiques du sexe masculin et donc à un phénotype ambigu (figure $3 E$ ).

Les méthodes pour découvrir ces nouveaux gènes sont simples en théorie mais plus difficiles à mettre 


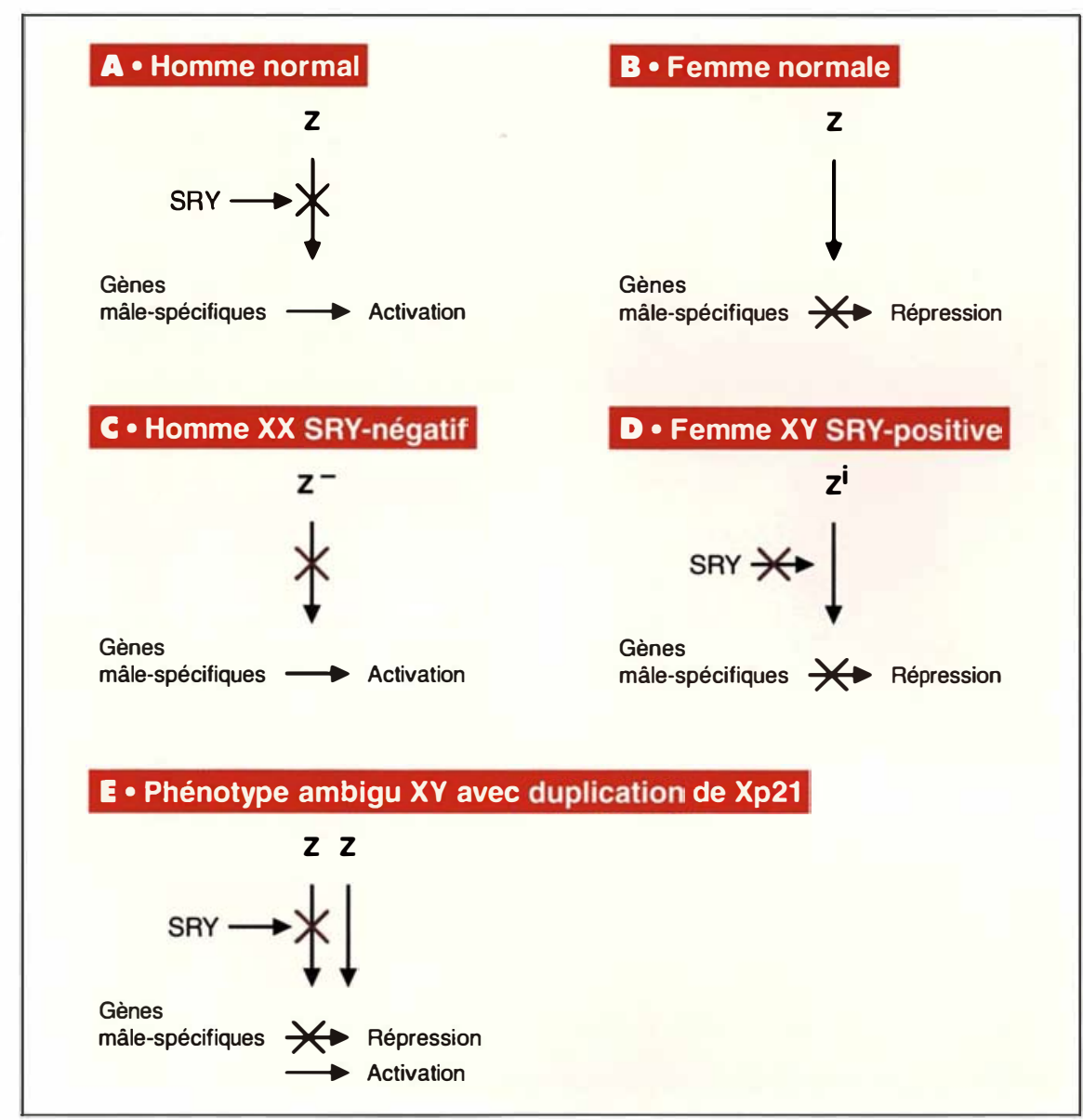

Figure 3. Modèle de cascade de régulation du déterminisme du sexe chez les mammifères. Ce modèle soutient l'hypothèse selon laquelle SRY aurait pour rôle d'inhiber l'action d'un gène $\mathrm{Z}$, lui-même répresseur des gènes spécifiques du sexe masculin. Chez un homme normal ou un homme $X X$ porteur de SRY, SRY inhibe Z, permettant ainsi une activation des gènes spécifiques du sexe masculin (A). En revanche, chez une femme normale, ou $X Y$ avec une délétion de SRY, Z peut réprimer la voie masculine en l'absence de SRY (B). Une perte de fonction totale ou partielle de Z peut expliquer l'apparition d'un phénotype masculin plus ou moins ambigu chez un individu $X X(C)$. Au contraire, si une mutation affecte la relation SRY-Z pour rendre $\mathrm{Z}$ indépendant ou insensible à l'action de SRY, l'effet dominant du chromosome $Y$ sur la détermination du testicule est court-circuité (mutation $Z^{i}, D$ ). Une duplication d'une région critique en $X p 21-22$ aboutissant à une réversion sexuelle, il est possible que $\mathrm{Z}$ soit localisé dans cette région et que, étant en double dose, il ne puisse subir une complète répression par SRY. Cela conduirait à une activation seulement partielle des gènes spécifiques du sexe masculin et donc à un phénotype ambigu (E). en pratique. Les patients, à la fois atteints d'ambiguïé sexuelle et porteurs d'une anomalie chromosomique détectable en cytogénétique, sont une source potentielle d'informations. La corrélation génotypephénotype permet d'attirer l'attention sur des régions du génome qui, lorsqu'elles sont délétées, réarrangées ou déplacées vers un autre locus, donnent naissance à une réversion de sexe. Cette méthode est limitée par la difficulté d'identifier précisément les points de cassure, ainsi que par la variabilité phénotypique présentée par ce type de patients. L'analyse de liaison est une stratégie qui pourrait permettre la caractérisation de régions impliquées dans le déterminisme du sexe. Mais cette méthodologie requiert l'analyse de cas familiaux qui sont très rares dans l'espèce humaine. Les modèles animaux fournissent une alternative intéressante. Certains gènes ou loci génétiques sont déjà caractérisés chez la souris et la recherche de leurs homologues humains pourrait être une méthode efficace pour identifier de nouvelles étapes de la cascade de détermination sexuelle. Le porc est également une espèce particulièrement intéressante par la fréquence élevée (jusqu'à $1 \%$ ) d'hermaphrodites observés dans les élevages. Chez la chèvre, une mutation dite polled a été observée, qui associe l'absence de cornes à un hermaphrodisme.

\section{Les autres gènes du déterminisme du sexe}

Certains exemples de gènes, loci ou régions génomiques jouant potentiellement un rôle dans le déterminisme sexuel chez les mammifères sont présentés. Cette liste est bien sûr incomplète mais elle met surtout l'accent sur les gènes candidats les plus intéressants (Tableau II).

\section{Le gène $W T 1$}

Plusieurs maladies humaines associent des anomalies rénales à des anomalies du développement sexuel. Les patients atteints du syndrome de Denys-Drash présentent une susceptibilité accrue à l'apparition d'une tumeur de Wilms et un pseudohermaphrodisme. Le syndrome WAGR désigne des individus présentant une 


\section{RÉFÉRENCES}

28. Tommerup N, Schempp W, Meinecke P Pedersen S, Bolund L, Brandt C, Goodpasture C, Guldberg P, Held KR, Reinwein $H$ Saugstad OD, Scherer G, Skjeldal O, Toder $R$, Westvik J, van der Hagen CB, Wolf U. As signment of an autosomal sex reversal locus (SRA1) and campomyelic dysplasia (CMPD1) to $17 \mathrm{q} 24.3-\mathrm{q} 25.1$. Nature Genet $1993 ; 4: 170$ 3.

29. Bardoni B, Floridia G, Guioli S, Peverali $G$, Anichini $C$, Cisternino $M$, Casalone $R$, Danesino C, Fraccaro M, Zuffardi O, Camerino G. Functional disomy of Xp22-pter in three males carrying a portion of $X_{p}$ translocated to Yq. Hum Genet 1993 ; 91 : 333-8.

30. Arn P, Chen H, Tuck-Muller CM, Mankinen C, Wachtel G, Li S, Shen CC, Wachtel SS. SRVX, a sex reversing locus in Xp21.2 p22.11. Hum Genet 1994 ; 93 : 389-93.

31. Bardoni B, Zanaria E, Guioli S, Floridia G, Worley KC, Tonini G, Ferrante E, Chiumello G, McCabe ERB, Fraccaro M, Zuffardi $O$, Camerino $G$. A dosage sensitive locus at chromosome Xp21 is involved in male to female sex reversal. Nature Genet $1994 ; 7$. 497-501.

32. Bennett CP, Docherty Z, Robb SA, Ramani P, Hawkins JR, Grant D. Deletion 9p and sex reversal. $\int$ Med Genet $1993 ; 30$ : 518 20.

33. Walker AP, Bocian M. Partial duplication 8q12-q21.2 in two sibs with maternally derived insertional and reciprocal translocations: case reports and review of partial duplications of chromosome 8. Am JMed Genet $1987 ; 27: 3-22$

34. Wilkie AOM, Campbell FM, Daubeney P, Grant DB, Daniels RJ, Mullarkey M, Affara NA, Fitchett M, Huson SM. Complete and partial $X Y$ sex reversal associated with terminal deletion of 10q: report of 2 cases and literature review. Am I Med Genet 1993 ; $46: 597-600$

35. McElreavey $\mathrm{K}$, Vilain E, Cotinot $\mathrm{C}$, Payen E, Fellous M. Control of sex determination in animals. Eur J Biochem 1993 ; 218 : 769-83.

36. Vilain E, Le Fiblec B, Morichon-Delvallez N, Brauner R, Dommergues $M$, Dumez Y, Jaubert F, Boucekkine C, McElreavey K, Vekemans M, Fellous M. $S R Y$-negative XX fetus with complete male phenotype. Lancet $1994 ; 343: 240-1$

37. Payen EJ, Cotinot CY. Sequence evolution of $S R Y$ gene within Bovidae family. Mamm Genome 1995 (sous presse)

38. Foster JW, Dominguez-Steglich A, Guioli S, Kwok C, Weller PA, Stevanovic M, Weissenbach I, Mansour S, Young ID, Goodfellow PN, Brook JD, Schafer A\}. Campomelic dysplasia and autosomal sex reversal caused by mutations in an $S R Y$-related gene. Nature 1994 ; 372: 525-30.

39. Zanaria E, Muscatelli F, Bardoni B, Stoom TM, Guioli S, et al. (14 auteurs). An unusual member of the nuclear hormone receptor superfamily responsible for X-linked adrenal hypoplasia congenita. Nature tumeur de Wilms, une aniridie, des anomalies urogénitales et un retard mental. Dans ces deux cas, le gène suppresseur de tumeur WT1 a été mis en cause $[15,16]$. Comme son homologue humain, la protéine codée par le gène murin wtl possède une structure particulière en doigts de zinc qui lui permet d'agir comme facteur de transcription. Son expression commence dès le neuvième jour du développement embryonnaire au niveau du rein en formation et de la gonade primitive. Les souris transgéniques homozygotes pour une mutation dans wtl meurent in utero avant le quinzième jour de développement [17]. Elles présentent une absence de reins, des anomalies cardiaques et pulmonaires. Bien que l'absence de wtl ne semble pas affecter la survie des cellules germinales, ces souris ne présentent pas de gonade du fait d'un défaut de formation et de maintien de la crête génitale. Ce phénotype est à rapprocher des patientes $\mathrm{XY}$ atteintes du syndrome de Denys-Drash qui possèdent des gonades fibreuses dépourvues de structures différenciées et de cellules germinales. Ces expériences tendent à prouver que les gènes $W T 1$ et wtl sont indispensables au développement rénal et gonadique, par induction du développement des structures primaires de ces organes.

\section{Ftz-F1}

Les facteurs SF1 (steroidogenic factor 1 ou orphan nuclear receptor) et ELP (embryonal long terminal repeat-binding protein) sont deux protéines issues d'un usage alternatif de sites d'épissage et de promoteurs d'un même gène murin nommé FTZ-F1 en raison de son homologie avec un facteur régulateur de l'homéogène de drosophile fushi tarazu [18]. La protéine ELP, exprimée très précocement dans l'embryon, a été identifiée à partir de cellules de carcinome embryonnaire mais son rôle reste inconnu. Les premiers transcrits de SFl sont détectés au $9^{\mathrm{e}}$ jour du développement embryonnaire de la souris dans la gonade indifférenciée [19]. L'expression est stable dans le testicule jusqu'après la naissance au niveau des cellules de Leydig et de Sertoli. La présence de sites de fixation de SFl sur tous les promoteurs de gènes codant pour les cytochromes P450 stéroïdogéniques suggère fortement un rôle clé de SFl dans la régulation de la stéroïdogenèse. Des souris transgéniques dont le gène SFl est muté par recombinaison homologue ont été créées pour confirmer cette hypothèse $\left(\mathrm{m} / \mathrm{s} n^{\circ} 10\right.$, vol. 10, p. 1055) [20-22]. Les souris qui n'expriment pas SF1 meurent peu après la naissance d'une insuffisance surrénalienne. L'autopsie de ces animaux a révélé l'absence totale de glandes surrénales et de gonades. Cette agénésie gonadique et, par conséquent, l'absence de production d'hormones sexuelles a pour corollaire un développement féminin avec structures müllériennes de tous les animaux, quel que soit leur sexe chromosomique. Au jour 12 du développement, les cellules de la crête génitale des animaux dépourvus de SF1 présentent un aspect caractéristique de cellules subissant l'apoptose et finissent par disparaître. L'absence de SFl ne semble pas affecter directement la survie des cellules germinales qui dégénèrent cependant par la suite, faute de trouver les structures nécessaires à leur développement. L'expression précoce de SFl dans les cellules de Sertoli fœtales ainsi que le phénotype drastique des souris complètement dépourvues de SF1 suggèrent que cette protéine joue de multiples rôles dans la différenciation gonadique et qu'elle est indispensable pour le développement des gonades et des glandes surrénales. Il a été démontré que $\mathrm{SF} 1$ se liait à une séquence spécifique dans le promoteur de l'AMH et qu'il permettait son activation grâce à un ligand qui reste inconnu. L'homologue humain de SF1 n'est pas identifié.

Les gènes $W T 1$ et $F T Z-F 1$, antérieurs à $S R Y$ dans la cascade, ne semblent pas intervenir directement dans le déterminisme sexuel des gonades, mais plutôt dans l'existence même des ébauches gonadiques, celles-ci étant nécessaires à l'expression de $S R Y$. Il serait néanmoins intéressant de vérifier si ces gènes peuvent avoir une influence sur l'expression de $S R Y$.

\section{L'hormone antimüllérienne}

L'hormone antimüllérienne ou AMH est une glycoprotéine sécrétée par les cellules de Sertoli dans le testicule 
Tableau II

TABLEAU RÉCAPITULATIF DES DIFFÉRENTS GĖNES CANDIDATS POUR JOUER UN RÔLE DANS LA CASCADE DE DÉTERMINISME DU SEXE CHEZ LES MAMMIFĖRES

\begin{tabular}{|c|c|c|c|c|c|}
\hline Gènes & Espèce & Localisation & Famille & Fonction & Phénotypes des mutations \\
\hline$F t z-F 1$ & souris & $?$ & récepteur nucléaire & $\begin{array}{c}\text { facteur de transcription, } \\
\text { activateur? }\end{array}$ & $\begin{array}{l}\text { agénésie des gonades } \\
\text { et des surrénales }\end{array}$ \\
\hline $\begin{array}{l}\text { WT1 } \\
\text { wt1 }\end{array}$ & $\begin{array}{l}\text { homme } \\
\text { souris }\end{array}$ & $\begin{array}{l}11 \mathrm{p} 13 \\
?\end{array}$ & $\begin{array}{l}\text { protéines à doigts } \\
\text { de zinc }\end{array}$ & facteur de transcription & $\begin{array}{c}\text { femme } X Y \text {, dysgénésie } \\
\text { gonadique, WAGR, Denys-Drash }\end{array}$ \\
\hline $\begin{array}{l}\text { SRY } \\
\text { sry }\end{array}$ & $\begin{array}{l}\text { homme } \\
\text { souris }\end{array}$ & $\begin{array}{l}Y p \\
Y p\end{array}$ & $\begin{array}{l}\text { protéines à high } \\
\text { mobility group }\end{array}$ & $\begin{array}{l}\text { facteur de transcription, } \\
\text { répresseur? }\end{array}$ & $\begin{array}{c}\text { femme } X Y \text {, dysgénésie } \\
\text { gonadique }\end{array}$ \\
\hline MIS & homme & chr 19 & $\begin{array}{l}\text { glycoprotéine } \\
\text { TGF } \beta \text {-like }\end{array}$ & $\begin{array}{l}\text { hormone de régression } \\
\text { des canaux de Muller }\end{array}$ & $\begin{array}{l}\text { homme avec persistance } \\
\text { des canaux }\end{array}$ \\
\hline $\begin{array}{l}\text { SRA1 lié } \\
\text { à CMD1 }\end{array}$ & homme & $17 q 24$ & $?$ & $?$ & $\begin{array}{c}\text { réversion de sexe, } \\
\text { dysplasie campomyélique }\end{array}$ \\
\hline SRVX & homme & $X p 22$ & $?$ & $?$ & $\begin{array}{l}\text { réversion de sexe, } \\
\text { ambiguïtés génitales }\end{array}$ \\
\hline$?$ & homme & $\operatorname{chr} 8,9,10 \ldots$ & $\ldots ?$ & $?$ & $\begin{array}{l}\text { réversion de sexe, ambiguïtés } \\
\text { génitales, pseudohermaphrodisme }\end{array}$ \\
\hline
\end{tabular}

fotal et adulte et par les cellules de la granulosa dans l'ovaire postnatal $[23,24]$. Chez le mâle, elle est responsable de la régression des canaux de Müller qui sont les ébauches embryonnaires de l'utérus, des oviductes et de la partie supérieure du vagin. Des mutations dans le gène de l'AMH ont été identifiées dans des patients présentant un syndrome de persistance des canaux de Müller [25]. Bien que pourvus d'un utérus, d'oviductes et d'un vagin, ces hommes ont un développement testiculaire normal. Chez des souris femelles transgéniques [26], l'AMH induit la régression des canaux de Müller et une réduction notable du nombre de cellules germinales conduisant à leur disparition totale quelques jours après la naissance. Des structures similaires à des tubes séminifères sont également observées dans leurs ovaires comme dans des ovaires de rates traitées in vitro par l'AMH. Ces observations, ainsi que la persistance de l'expression de l'AMH après la période de régression des canaux de Müller chez le mâle, suggèrent une multiplicité de rôles dans la différenciation sexuelle.

Bien qu'une séquence potentielle de fixation de SRY ait été identifiée dans $\mathrm{m} / \mathrm{s} n^{\circ} 4$, vol. 11 , avril 95 le promoteur de l'AMH, on peut néanmoins exclure l'AMH comme étant la cible directe de SRY. Les profils temporels d'expression des deux protéines sont décalés et l'AMH, au contraire de SRY, ne semble pas indispensable pour un développement testiculaire normal. Il doit donc exister des étapes intermédiaires entre SRY et l'AMH dans la cascade $(\mathrm{m} / \mathrm{s}$ $n^{\circ} 2$, vol. 11, p. 300).

\section{Le locus de la dysplasie campomé- lique}

La dysplasie campomélique est une maladie autosomique récessive associée à des anomalies, squelettiques principalement. Une forte proportion de ces patients XY présente également une réversion de sexe [27]. Un locus a récemment été identifié en $17 q 24$, entre les gènes de l'hormone de croissance et de la thymidine kinase $1\left(m / s n^{\circ} 2\right.$, vol. $11, p .300$ [28]).

\section{Région Xp21-22}

Certains patients porteurs d'un réarrangement d'origine paternelle où un fragment de Xp est transloqué en Yq, présentent, outre des symptômes malformatifs, des ambiguités sexuel- les ou une hypoplasie des organes génitaux [29]. L'analyse cytogénétique et moléculaire a montré des points de cassure différents dans les cinq cas publiés, suggérant que le phénotype est plus probablement dû à un dosage anormal de gène(s) situés dans la région Xp21-Xpter qu'à la cassure d'un gène. Cette hypothèse est soutenue par l'existence de plusieurs patients XY présentant des anomalies sexuelles comparables et porteurs d'une duplication partielle du bras court de leur chromosome X. Il semble donc qu'un double dosage de gène(s) localisés en Xp21-22 et baptisés dans un premier temps SRVX (sex reversal $X)$ [30] interfère avec un développement masculin normal (figure $3, E)$. Une région de $160 \mathrm{~kb}$, critique pour la réversion sexuelle $\mathrm{XY}$, vient d'être localisée en Xp21 [31]. Cette région contiendrait un gène, désormais appelé DSS (dosage sensitive sex reversal), dont le double dosage causerait une réversion de sexe des individus XY $\left(\mathrm{m} / \mathrm{s} n^{\circ} 11\right.$, vol. 10, p. 1170).

Anomalies chromosomiques et réversion de sexe

L'analyse cytogénétique de patients présentant des ambiguïtés sexuelles a 
permis de mettre en évidence certaines régions du génome. Cing cas de réversion de sexe chez des individus XY ont été corrélés à des réarrangements impliquant la bande 9p24 [32]. Des patients présentant des ambiguïtés génitales et porteurs de réarrangements de régions des chromosomes 8 et 10 ont également été décrits [33, 34]. Certains de nos patients présentent des anomalies cliniques et cytogénétiques semblables et nous laissent supposer la présence de gènes importants dans ces régions.

\section{Conclusion}

S'il est maintenant clairement établi que $S R Y$ est l'initiateur majeur de la détermination sexuelle, de nombreux autres gènes sont également impliqués dans cette voie et restent à être identifiés. Dans ce domaine, nos connaissances ont, chez les mammifères, un retard considérable par rapport aux modèles animaux que sont la mouche $D$. melanogaster ou le nématode C. elegans [35]. Chez ces deux espèces, comme probablement chez les mammifères, la détermination sexuelle utilise une stratégie commune à de nombreux processus de développement, impliquant une hiérarchie ordonnée de gènes de détermination, avec des régulations positives ou négatives en cascade. Plus étonnante est l'absence de conserva- tion des mécanismes moléculaires, malgré une stratégie similaire. Non seulement les molécules impliquées dans la détermination sexuelle ne sont pas conservées entre les différents organismes étudiés, mais SRY a également une séquence variable parmi les mammifères (surtout hors de la boîte HMG) et semble avoir une vitesse d'évolution particulièrement rapide chez certaines espèces (m/s $n^{\circ} 10$, vol. 9, p. 1137).

L'élucidation de toutes les étapes de la détermination sexuelle dans l'espèce humaine permettra peut-être de mieux comprendre les mécanismes en cause dans les maladies de la détermination du sexe. De nombreuses questions restent en effet non résolues pour le moment. Comment expliquer la variabilité phénotypique des mâles XX et des hermaphrodites vrais XX ? Comment expliquer l'absence de cellules germinales à l'âge adulte chez les mâles XX, alors qu'au stade fotal des cellules germinales peuvent être observées [36] ? Comment expliquer la fréquence élevée de gonadoblastome (une tumeur germinale) chez les femmes XY avec dysgénésie gonadique ? Ces questions font appel à des domaines connexes à la détermination sexuelle comme la spermatogenèse ou les communications intercellulaires et nécessiteront probablement une compréhension globale des phénomènes survenant dans la gonade en développement

\section{Summary}

A view on sex determination in mammals

Sex determination is the set of mechanisms that lead a fetal indifferenciated gonad to commit into the development of either a testis or an ovary. This pathway is a very complex developmental cascade that requires interaction of many genes. The $S R Y$ gene (sex determining region, Y chromosome) has been identified as being the testis determining factor which is the primary signal triggering testis formation. Thanks to the analysis of sex reversed patients or animal models, some other genes are shown to play a role in sex determination. However, many steps of the cascade are unknown and remain to be identified.

\section{Remerciements}

Les auteurs remercient Catherine Alcaide pour ses critiques et conseils pendant la préparation de ce manuscrit.

\section{TIRÉS À PART}

S. Barbaux.

Note ajoutée aux épreuves

Depuis la rédaction de cet article, le gène responsable de la dysplasie campomélique a été identifié. Il s'agit d'un gène de la famille des facteurs de transcriptions SOX, nommé SOX9 [38].

Le gène $D A X-1$, codant pour une protéine de la famille des récepteurs hormonaux nucléaires, a été cloné. Il est responsable de l'hypoplasie congénitale des surrénales et est localisé dans l'intervalle minimal en Xp21 pour le locus DSS. Il apparaît donc comme un candidat potentiel pour ce gène DSS [39]. 